\title{
Event-related theta power increases in the human EEG during online sentence processing
}

\author{
Marcel C.M. Bastiaansen ${ }^{a, *}$, Jos J.A. van Berkum ${ }^{a-c}$, Peter Hagoort ${ }^{a, c}$ \\ ${ }^{a}$ Max Planck Institute for Psycholinguistics, P.O. Box 310, 6500 AH Nijmegen, The Netherlands \\ ${ }^{b}$ Department of Psychology, University of Amsterdam, Roetersstraat 15, 1018 WB Amsterdam, The Netherlands \\ ${ }^{c}$ F.C. Donders Centre for Cognitive Neuroimaging, P.O. Box 9101, 6500 HB Nijmegen, The Netherlands
}

Received 14 July 2001; received in revised form 12 December 2001; accepted 12 December 2001

\begin{abstract}
By analyzing event-related changes in induced band power in narrow frequency bands of the human electroencephalograph, the present paper explores a possible functional role of the alpha and theta rhythms during the processing of words and of sentences. The results show a phasic power increase in the theta frequency range, together with a phasic power decrease in the alpha frequency range, following the presentation of words in a sentence. These effects may be related to word processing, either lexical or in relation to sentence context. Most importantly, there is a slow and highly frequency-specific increase in theta power as a sentence unfolds, possibly related to the formation of an episodic memory trace, or to incremental verbal working memory load. (c) 2002 Elsevier Science Ireland Ltd. All rights reserved.
\end{abstract}

Keywords: Induced band power; Event-related desynchronization; Electroencephalograph rhythms; Theta rhythm; Alpha rhythm; Sentence processing; Episodic memory; Working memory

The event-related reactivity of rhythmic electroencephalographic (EEG) activity can be analyzed, e.g. through the computation of induced band power (IBP) changes $[4,5,10]$. Using the IBP technique, it has been shown that the theta rhythm of the human, scalp-recorded EEG shows eventrelated increases in power during memory encoding and retrieval [4], and during the recognition of words and faces [1]. This reactivity has been shown to be highly frequency-specific, since it has commonly been observed in the theta, but not in the alpha frequency range. Spatially distributed, yet functionally coherent cell assemblies have been proposed to underly the representation of words in the brain [11]. Several authors have related the theta rhythm to the dynamics involved in cell assembly formation $[8,13]$. Therefore, one would expect to find event-related changes in theta during the processing of words. The first aim of the present paper is to verify this prediction.

In order to understand language, one has to form temporary representations of the incoming language material. Such temporary representations obviously require some form of working memory. It has previously been shown that

* Corresponding author. Tel.: +31-24-352-1911; fax: +31-24352-1213.

E-mail address: marcel.bastiaansen @mpi.nl (M.C.M. Bastiaansen). increases in theta power can be observed during tasks which engage verbal working memory processes $[3,6]$. The second aim of this paper is to explore whether eventrelated changes in theta power can be found during the processing of sentences, a task which is likely to engage verbal working memory.

Eighteen right-handed native Dutch undergraduate students (five males; age range, 19-25; mean age, 21 years), recruited from our local subject pool, participated in the experiment. None had any neurological impairment, had experienced any neurological trauma, or used neuroleptics. They were informed about the purpose and nature of the experiment, and gave their informed consent.

The stimulus material consisted of 450 Dutch sentences. Of these, 123 contained a syntactic violation, 30 contained a semantic violation, 121 contained a complex pronominal reference to an earlier introduced proper name, and 176 sentences were correct Dutch sentences. Every sentence contained 12 words at most ( 8.9 on average), with every word containing at most 12 letters. For the present analyses, only correct sentences which contained nine words or more were used. There were 150 such sentences. The IBP analyses were performed on the first eight words of these sentences. This avoids the inclusion of sentence-final words in the analyses, which may be a special case due to sentence wrap-up effects. 
To avoid eye movements, sentences were displayed word by word at a fixed rate, with every word shown in the centre of the screen. Each trial consisted of a $300 \mathrm{~ms}$ auditory warning tone, followed by a $650 \mathrm{~ms}$ blank screen, after which the sentence was presented, with every word displayed for $300 \mathrm{~ms}$ and a $300 \mathrm{~ms}$ blank screen between words. Subjects were instructed to read the sentences attentively. No additional task demands were imposed. After a short practice, the trials were presented in five blocks of about $14 \mathrm{~min}$, separated by rest periods.

The EEG was recorded from $29 \mathrm{Ag}-\mathrm{AgCl}$ scalp electrodes (see Fig. 3), against a left mastoid reference, with an $8 \mathrm{~s}$ time constant and a $30 \mathrm{~Hz}$ cut-off frequency, and was continuously sampled at $200 \mathrm{~Hz}$, with a 12-bit A/D resolution. The EEG was screened off-line for artefacts in an interval that ranged from $500 \mathrm{~ms}$ before to $4800 \mathrm{~ms}$ after sentence onset (that is, $600 \mathrm{~ms}$ after onset of the 8th word). Trials containing artefacts were rejected (16\% on average).

For the computation of the IBP changes, we followed the approach outlined by Klimesch [4]. IBP is computed in individually adjusted narrow frequency bands, based on the individual alpha peak frequency (IAF). The IAF $(M=10.32 \mathrm{~Hz}, \mathrm{SD}=0.782 \mathrm{~Hz})$ was determined for each subject separately, through a spectral analysis on the EEG segments ranging from $500 \mathrm{~ms}$ preceding sentence onset to $4800 \mathrm{~ms}$ after sentence onset. The EEG data were filtered with an FFT filter in four different frequency bands: theta (IAF $-6 \mathrm{~Hz}$ to IAF $-4 \mathrm{~Hz}$ ); lower- 1 alpha (IAF $-4 \mathrm{~Hz}$ to IAF $-2 \mathrm{~Hz}$ ); lower-2 alpha (IAF $-2 \mathrm{~Hz}$ to IAF); and upper alpha (IAF to IAF $+2 \mathrm{~Hz}$ ). Next, for each frequency band, the data were averaged, and the resulting (filtered) eventrelated potential (ERP) was subtracted from each (filtered) single trial in order to avoid a contamination of the IBP measures with the ERPs (see [4,5]). On the single-trial data thus obtained, a Hilbert transform was applied, which yields the envelope of the signal. The single-trial signal envelopes were averaged over trials, and IBP was computed as the percentage power increase or decrease in a particular frequency band in the first $600 \mathrm{~ms}$ interval following the onset of each word, relative to a reference interval from 300 to $0 \mathrm{~ms}$ preceding word onset (word-level effects), or as the percentage power increase or decrease in a particular frequency band in the first $4800 \mathrm{~ms}$ of sentence presentation, relative to a $500 \mathrm{~ms}$ pre-sentence reference interval (sentence-level effects).

The presentation of each word in a sentence elicits phasic changes in IBP in each of the four frequency bands studied (Fig. 1). In the theta and lower-1 alpha bands, there is a phasic IBP increase following word presentation which peaks earlier in the lower- 1 alpha band than in the theta band. In the lower- 2 and upper alpha bands, there is an initial, very early IBP increase which starts to develop before the word is actually presented, followed by an IBP decrease which coincides in time with the IBP increase in the lower bands. Table 1 lists the mean peak magnitudes and latencies of the phasic IBP changes for each frequency band, averaged over word positions and electrodes.

Single sample $t$-tests showed that the IBP changes (averaged over all electrodes, in a window around the peak latency; Table 1, right-hand column for the respective time windows) differed significantly from zero (all $P$ values $<0.05)$. In order to test for the scalp distribution of the IBP changes, analyses of variance (ANOVAs) for repeated measures were performed on the mean percentage IBP changes in a window (given in Table 1, right-hand column) around the peak latency, with Electrode (29 levels) as a

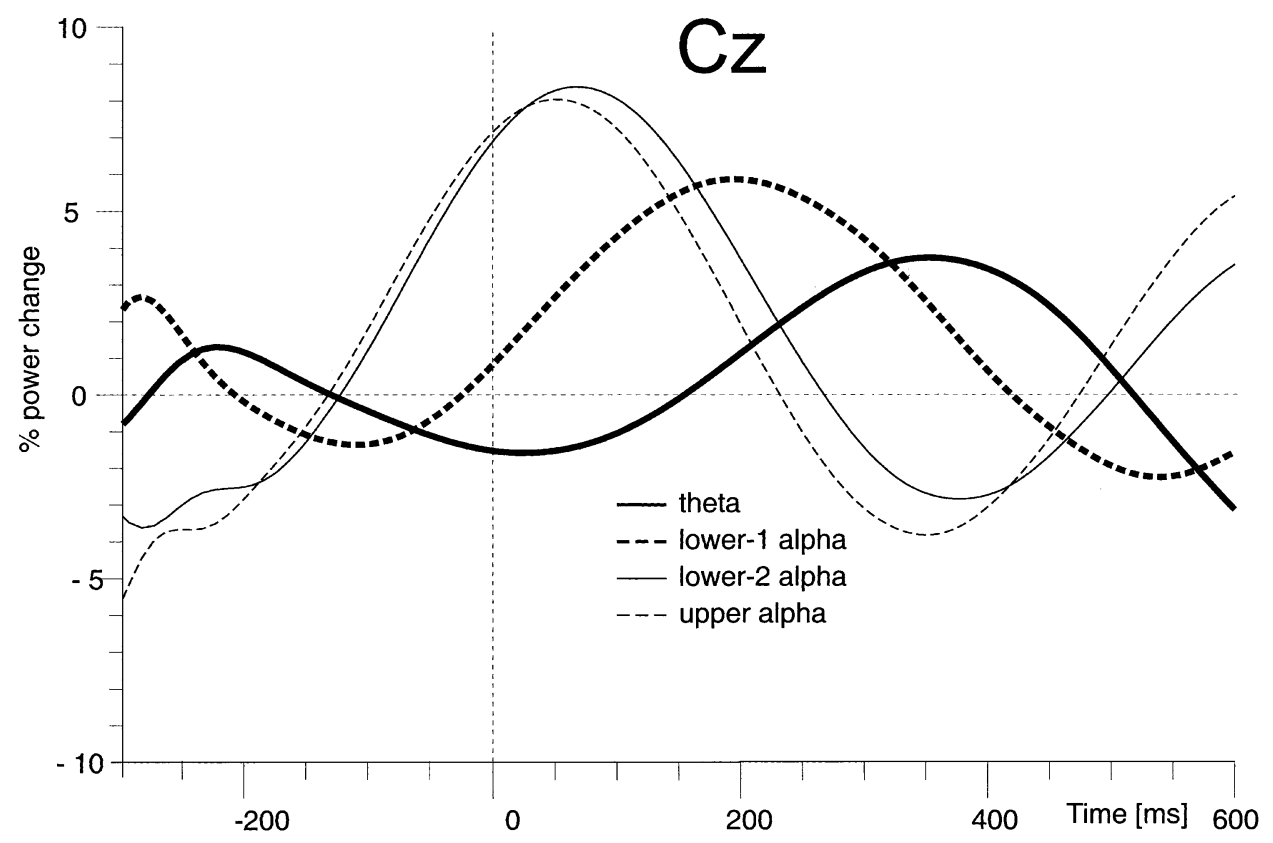

Fig. 1. Grand average temporal evolution of IBP changes in each of the frequency bands, averaged over word positions, for electrode Cz. 
Table 1

Grand average peak latency of IBP changes $^{a}$, and amplitude of IBP changes ${ }^{b}$ in a window around the peak latency in each of the frequency bands, averaged over word positions and electrodes ${ }^{d}$

\begin{tabular}{lccc}
\hline Measure & IBP peak latency $(\mathrm{ms})$ & IBP amplitude (\% change) & $\begin{array}{c}\text { Latency window used for } \\
\text { amplitude measures (ms) }\end{array}$ \\
Frequency band & Mean (SD) & Mean (SD) & $222-422$ \\
\hline Theta increase & $322(43)$ & $6.4(3.15)$ & $112-312$ \\
$\begin{array}{l}\text { Lower-1 alpha increase } \\
\text { Lower-2 alpha }\end{array}$ & $212(69)$ & $15.9(2.68)$ & $0-84$ \\
$\quad$ Early increase & $84(25)$ & $19.25(2.40)$ & $288-488$ \\
$\quad$ Late decrease & $388(26)$ & $-4.0(3.25)$ & $0-38$ \\
Upper alpha & $38(18)$ & $13.1(3.85)$ & $233-433$ \\
$\quad$ Early increase & $333(48)$ & $-7.0(3.46)$ & \\
$\quad$ Late decrease & &
\end{tabular}

a Left-hand column.

${ }^{b}$ Middle column.

${ }^{\mathrm{c}}$ Right-hand column.

${ }^{d}$ For most effects, a 200 ms window around the peak latency is used. However, for the early power increases in the lower-2 and upper alpha bands, this would have resulted in a pre-word onset of the window. In these cases, we defined the onset of the window at $t=0$.

repeated measures factor. Greenhouse-Geisser corrected $P$ values are reported in order to control for deviations from sphericity. ANOVAs were performed for each frequency band; for the lower-2 and upper alpha bands, these analyses were performed both on the early IBP increase and on the late IBP decrease. These analyses show that the IBP increases in the theta and lower-1 alpha, as well as the late IBP decreases in the lower-2 and upper alpha bands are widely distributed, as indicated by an absence of a significant main effect of Electrode (all $P$ values $>0.1$ ). However, main effects of Electrode were present in the ANOVAs on the early IBP increases in the lower-2 $\left(F_{28,244}=3.89, P=0.029\right)$ and upper $\left(F_{28,244}=10.03\right.$, $P<0.001)$ alpha bands, and point to an occipital maximum of these effects.

The grand average IBP changes over the course of an entire sentence are presented in Fig. 2. Fig. 2 shows that, next to the aforementioned phasic changes following individual words in a sentence, one can observe a slow increase in IBP in the theta frequency band as the sentence unfolds, which does not appear to be present in the other frequency bands.

In order to verify this statistically, the IBP changes at individual electrodes were tested for the presence of a linear trend against an alpha level of 5\%, in each of the four frequency bands. The results of these analyses show that only in the theta band, significant linear trends are present at central and temporo-parietal leads, indicating a gradual increase in theta power as the sentence unfolds. Fig. 3 shows the electrodes displaying a significant linear trend in a topographical layout. In the higher frequency bands, significant linear trends were found only sporadically (lower-1 alpha: electrodes FC3, RT and RTP; lower-2 alpha: electrodes RT and RTP; upper alpha: none; see Fig. 3 for the position of the electrodes mentioned here).

The IBP responses to the presentation of individual words in a sentence show a clear pattern of results. In the theta and lower-1 alpha bands, a widespread, phasic power increase can be identified, while at the same time, in the higher frequency bands, lower-2 alpha and upper alpha, a widespread power decrease can be observed. Since the temporal resolution of the IBP method is not as good as that of ERP measures [2], the peak latencies of these effects should be taken only as a rough indication of the timing of the neurophysiological phenomena driving the IBP responses. Nonetheless, these latencies roughly fall in the range of wellknown ERP components related to language processing,

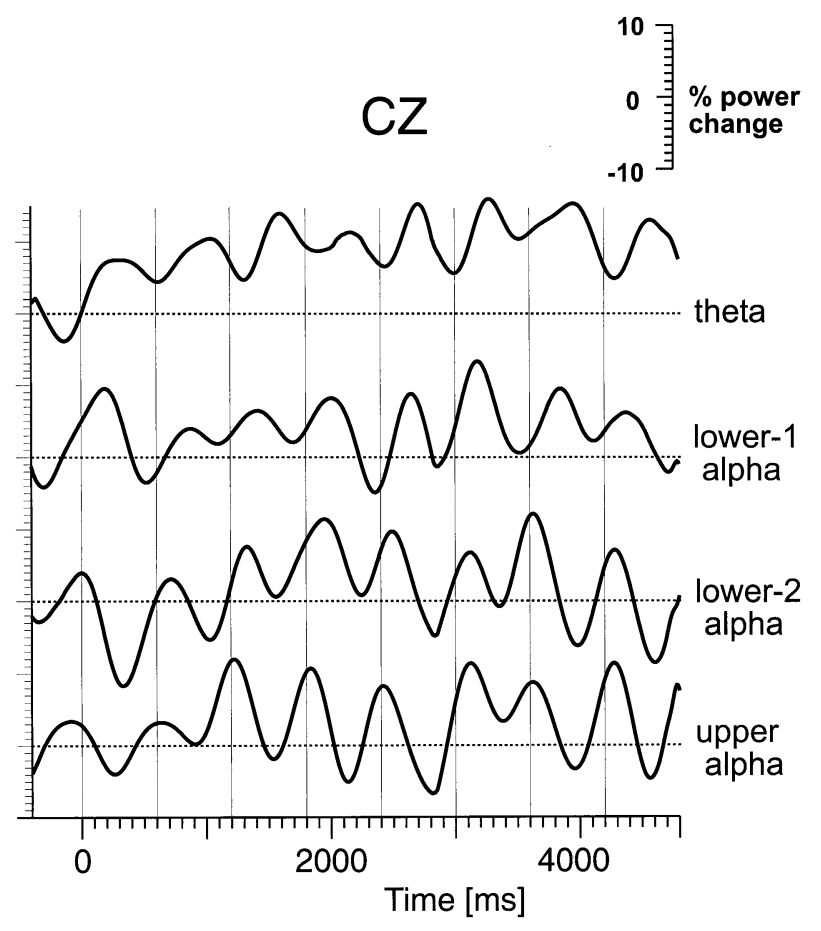

Fig. 2. Grand average temporal evolution of IBP changes during the presentation of the first eight words of a sentence, relative to a pre-sentence reference interval, for electrode $\mathrm{Cz}$. Note the slow IBP increase over time, which is present as a significant linear trend only in the theta band. 


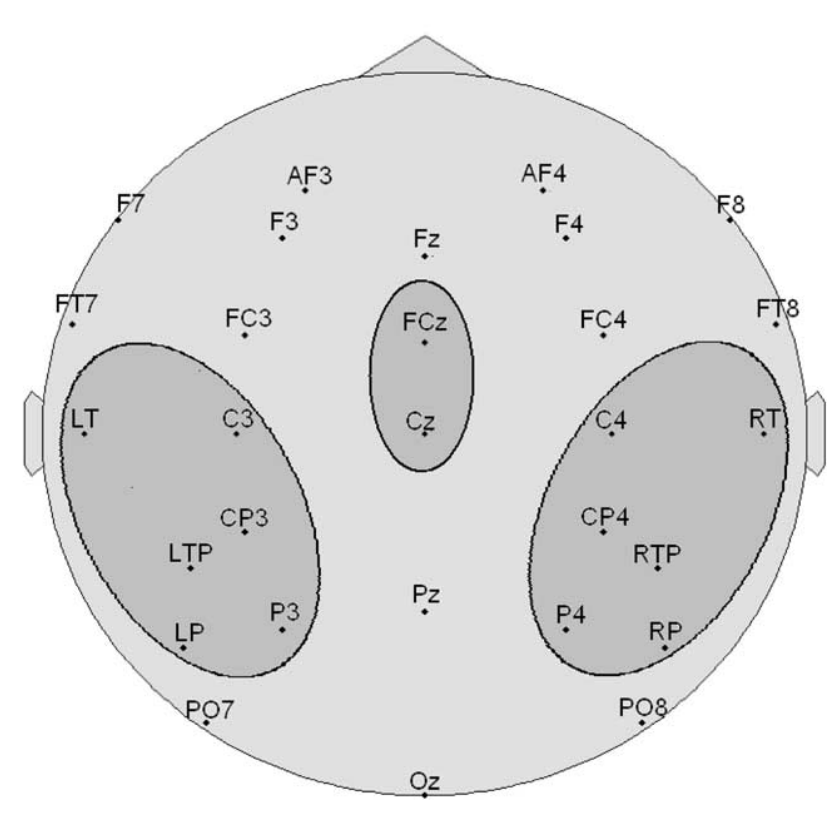

Fig. 3. Orthogonal projection of the electrode montage. The dark grey ovals contain electrodes which display a significant linear increase in theta power as the sentence unfolds.

such as the N400 [7] and the Left Anterior Negativity [9]. Therefore, it seems reasonable to hypothesize that the power increase in the lower frequency bands, and the concurrent power decrease in the higher bands, is related to some aspect of word processing, either lexical or in relation to sentence context. A possible candidate process underlying the phasic theta increase would be the activation of a cell assembly corresponding to the representation of the lexical item (see also [11]). Note also that the observed pattern of a power increase in the lower bands together with a power decrease in the higher bands is reminiscent of the pattern of results obtained during tasks engaging episodic memory [4] and word and face recognition [1].

Somewhat surprisingly, the (very) early power increases observed in the higher frequency bands start preceding word onset. One could argue that this pre-word onset may be related to anticipatory processes induced by the highly regular pace of the serial presentation. However, in our view, a more plausible interpretation is that this effect is caused by the fact that the reference interval (from 300 to $0 \mathrm{~ms}$ preceding word onset) coincides with the late power decrease in these bands: recovery of this decrease then yields an artefactual power increase.

The observation of a slow increase in theta power as the sentence unfolds is a most interesting observation. This effect is highly frequency-specific, since it reliably occurs only in the narrow $(2 \mathrm{~Hz})$ frequency band that we defined here as theta. Moreover, the slow theta increase displays a clear-cut scalp topography (Fig. 3). Since the theta rhythm has been related to episodic memory processes [12], this effect may reflect the formation of an episodic memory trace as the individual words in a sentence gradually converge into an overall understanding of the 'episode' described by the sentence. Alternatively, in view of the results relating theta increases to working memory $[3,6]$, the present slow theta increase might also be related to incremental verbal working memory load as the sentence unfolds. However, these interpretations are speculative, and may at best serve as working hypotheses, useful in guiding future research.

We would like to note in closing that generally, power increases are thought to reflect an increase in local synchrony. Therefore, synchronization between distant brain regions is not captured with the current methodology. Such processes can be better studied with methods such as coherence analysis (e.g. [14]), which would be a valuable complement to IBP analyses in general.

[1] Burgess, A.P. and Gruzelier, J.H., Short duration power changes in the EEG during recognition memory for words and faces, Psychophysiology, 37 (2000) 596-606.

[2] Clochon, P., Fontbonne, J.M., Lebrun, N. and Etévenon, P., A new method for quantifying EEG event-related desynchronization: amplitude envelope analysis, Electroenceph. clin. Neurophysiol., 98 (1996) 126-129.

[3] Gevins, A., Smith, M.E., McEvoy, L. and Yu, D., High-resolution EEG mapping of cortical activation related to working memory: effects of task difficulty, type of processing, and practise, Cereb. Cortex, 7 (1997) 374-385.

[4] Klimesch, W., EEG alpha and theta oscillations reflect cognitive and memory performance: a review and analysis, Brain Res. Rev., 29 (1999) 169-195.

[5] Klimesch, W., Russegger, H., Doppelmayr, M. and Pachinger, T., A method for the calculation of induced band power: implications for the significance of brain oscillations, Electroenceph. clin. Neurophysiol., 108 (1998) 123130.

[6] Krause, C.M., Sillanmäki, L., Koivisto, M., Saarela, C., Häggqvist, A., Laine, M. and Hämäläinen, H., The effects of memory load on event-related EEG desynchronization and synchronization, Clin. Neurophysiol., 111 (2000) 2071-2078.

[7] Kutas, M. and Hillyard, S.A., Reading senseless sentences: brain potentials reflect semantic incongruity, Science, 207 (1980) 203-205.

[8] Miller, R., Cortico-hippocampal Interplay and the Representation of Contexts in The Brain, Springer-Verlag, Berlin, 1991, $268 \mathrm{pp}$.

[9] Munte, T.F., Matzke, M. and Johannes, S., Brain activity associated with syntactic incongruencies in words and pseudowords, J. Cogn. Neurosci., 9 (1997) 300-311.

[10] Pfurtscheller, G. and Lopes da Silva, F.H., Event-related EEG-MEG synchronization and desynchronization: basic principles, Clin. Neurophysiol., 110 (1999) 1842-1857.

[11] Pulvermuller, F., Words in the brain's language, Behav. Brain Sci., 22 (1999) 253-336.

[12] Treves, A. and Rolls, E.T., Computational analysis of the role of the hippocampus in memory, Hippocampus, 4 (1994) 374-391.

[13] Von Stein, A. and Sarnthein, J., Different frequencies for different scales of cortical integration: from local gamma to long-range alpha/theta synchronization, Int. J. Psychophysiol., 38 (2000) 301-313.

[14] Weiss, S., Muller, H.M. and Rappelsberger, P., Theta synchronization predicts efficient memory encoding of concrete and abstract nouns, NeuroReport, 11 (2000) 23572361. 